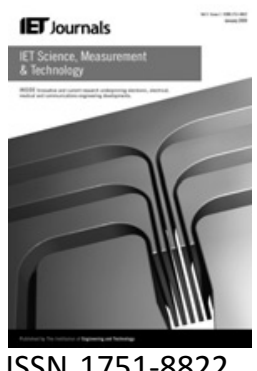

\title{
Ant Colony Optimisation-based radiation pattern manipulation algorithm for Electronically Steerable Array Radiator Antennas
}

\author{
J. Aelterman ${ }^{1, *}$ R. Goossens ${ }^{2} \quad$ F. Declercq ${ }^{2} \quad$ H. Rogier ${ }^{2}$ \\ ${ }^{1}$ Telecommunications and Information Processing Department, Ghent University, St.-Pietersnieuwstraat 41, 9000 Ghent, \\ Belgium \\ ${ }^{2}$ Information Technology Department, Ghent University, St.-Pietersnieuwstraat 41, 9000 Ghent, Belgium \\ *J. Aelterman was also working at the Information Technology Department when the work was carried out \\ E-mail: jan.aelterman@telin.ugent.be
}

Abstract: A new algorithm for manipulating the radiation pattern of Electronically Steerable Array Radiator Antennas is proposed. A continuous implementation of the Ant Colony Optimisation (ACO) technique calculates the optimal impedance values of reactances loading different parasitic radiators placed in a circle around a centre antenna. By proposing a method to obtain a suitable sampling frequency of the radiation pattern for use in the optimisation algorithm and by transforming the reactance search space into the search space of associated phases, special care was taken to create a fast and reliable implementation, resulting in an approach that is suitable for real-time implementation. The authors compare their approach to analytical techniques and optimisation algorithms for calculating these reactances. Results show that the method is able to calculate near-optimal solutions for gain optimisation and side lobe reduction.

\section{Introduction}

Since the advent of mobile communications, there has been an ever growing demand for more users and higher data rates. As it is infeasible to reserve larger parts of the already crowded electromagnetic spectrum for use in mobile communication systems, there is a growing interest in other techniques for maintaining or increasing the channel capacity between the user device and the base station within the limited available bandwidth. One of those techniques is the use of adaptive antenna systems. These systems use the combination of direction of arrival (DOA) estimation with adaptive beamforming techniques to create a truly 'intelligent' antenna system. New technologies, such as fourth generation of mobile communications (4G) or the 802.11n (WLAN) standard, will rely heavily on intelligent antenna systems.

Conventional steerable antenna arrays require amplitude and phase control circuitry together with an RF receiver at each antenna element, thereby resulting in a very complex and high-cost system, which is not suited for mass-produced consumer products or for integration into user terminals. In an Electronically Steerable Array Radiator Antenna (ESPAR) [1], a single RF receiver path is applied for the centre element only, and beamsteering results from electronically controlling the reactances that load the equally spaced parasitic radiators surrounding the active antenna element. The benefits in terms of reduced hardware complexity and cost come at the price of a more complex steering algorithm, since the loads of the parasitic elements influence the radiation pattern of the array through mutual coupling. Finding the correct reactances to achieve a desirable radiation pattern is a highly non-linear problem and an optimisation method, in a deterministic or stochastic way, is required to find the correct reactances. In [2] the authors circumvene difficulties by introducing an analytical approach to ESPAR beamsteering. However, this approach is restricted to control only the direction and magnitude of the main beam's radiation vector magnitude. For side lobe suppression, controlling the beamwidth or optimising directivity, which is 
a much more useful antenna property, more complex optimisation methods are required. Deterministic optimisation methods to find the appropriate reactances [3] are mainly based on the steepest gradient or oversimplification through performing the multivariate optimisation as successive univariate optimisation processes [4]. They often require perfect knowledge of the objective function and its derivatives and they are computationally complex, especially for multi-dimensional optimisation. Although they converge fast to a solution, they are very likely to converge to a local optimum and therefore these algorithms are sub-optimal. There exist other techniques, for example, random restarts, that introduce stochastic behaviour to these deterministic optimisation techniques. It can be argued that doing this transforms the deterministic algorithm into another ad hoc metaheuristic algorithm. A metaheuristic algorithm adapted to a specific application, for example, an Ant Colony Optimisation (ACO) [5] algorithm with application-specific pheromone operators or an Evolution Strategy (ES) [6] or Genetic Algorithm [7, 8] with recombination operators tailored to a specific application will obviously perform better. Knowledge of the application, and the search space, allows the designer of these application-specific metaheuristic algorithms to take steps against convergence to local optima. In this versatility lies the advantage of metaheuristic algorithms, such as the proposed ACO, over other methods when used in optimisation problems that are prohibitively complicated to solve analytically. The lack of a tailored optimisation algorithm for the beam pattern of ESPARs limits the number of parasitic antenna elements that can be steered, and hence the beamforming capabilities of the ESPAR. In this paper, a versatile radiation pattern manipulation strategy is followed. We rely on the continuous implementation of the ACO technique that calculates the optimal impedance values of the reactances loading the different parasitic radiators placed in a circle around a center antenna. By specially adapting the ACO scheme to the problem at hand, through phase domain steering (Section 4.1.1) and suitable sampling of the radiation pattern (Section 4.1.2), a fast and reliable beamforming technique is obtained, avoiding problems with convergence and resulting in an approach that is suitable for real-time implementation.

The paper is organised in the following way: In Section 2, we provide an overview of the mathematical model of an ESPAR, a uniform circular array (UCA) system driven by variable loads. This model is used in the optimisation algorithm, which is explained in Section 3. In Section 4, we demonstrate the algorithm on several optimisation problems. We use these examples to illustrate several implementation issues relevant to the ESPAR beamforming problem.

\section{Electromagnetic model of ESPAR}

Consider an antenna array, consisting of $N$ quarterwavelength monopole antennas uniformly distributed over a circle with radius $R$. Although in this paper we demonstrate the proposed beamforming approach using an UCA of monopole antennas, more complex array elements or array geometries are just as easily processable in the presented beamforming approach. In the centre of this UCA, a monopole element (antenna 0) with a standalone antenna impedance $Z_{00}$ is excited by an RF signal source with internal voltage $V_{0}$ and output impedance $Z_{s}$ (Fig. 1). Each antenna of the UCA is loaded with an adjustible reactive load $j x_{n}=1 /\left(j \omega C_{n}\right)(n=1, \ldots, N)$. It is our aim to determine the exact value of the reactive loads that satisfy the beamforming requirements, by means of an optimisation strategy based on the ACO technique.

The only driven antenna in the system of Fig. 1 is the centre one. Hence, the radiation pattern of the system will heavily depend on mutual coupling effects between this antenna and the parasitic radiators of the UCA. So, in order to use this configuration for beamforming, one needs to fully characterise the mutual coupling effects in the array so as to calculate the effect of the parasitic radiators on the radiation pattern of the array. The excited centre antenna element introduces currents $i_{n}(n=1, \ldots, N)$ in the antennas of the UCA through mutual coupling. Mutual coupling in the antenna array is described by the mutual coupling matrix $Z$. The elements $Z_{n k}$ describe the coupling between antenna $n$ and antenna $k(n, k=0, \ldots, N)$. This effect will alter the antenna impedance, potentially jeopardising matching requirements, this topic is covered in Section 4.2. For the centre element, the induced current $i_{0}$ is described by

$$
V_{0}=\left(Z_{s}+Z_{00}\right) i_{0}+\sum_{k=1}^{N} Z_{0 k} i_{k}
$$

whereas for the parasitic elements $(1 \leq n \leq N)$ the induced currents $i_{n}$ are described by

$$
0=\left(Z_{n n}+j x_{n}\right) i_{n}+\sum_{k=0, k \neq n}^{N} Z_{n k} i_{k}
$$

It is clear that in this description all antennas in the ESPAR couple with each other. It is possible to rearrange (1) and (2) resulting in

$$
\boldsymbol{I}=(\boldsymbol{Z}+j \boldsymbol{X})^{-1} \boldsymbol{V}
$$

where $\boldsymbol{I}=\left[i_{0}, i_{1}, i_{2}, \ldots, i_{N}\right]^{\mathrm{T}}$ is a current vector containing the currents flowing through the antenna terminals, $\boldsymbol{V}=\left[V_{0}, 0,0, \ldots, 0\right]^{\mathrm{T}}$ is the excitation vector of dimension $N+1$, which only has one non-zero component for the driven centre antenna. $Z$ is the impedance matrix containing the output impedance $Z_{s}$ of the element injecting the RF signal, as well as the antenna impedances $Z_{n n}$ on the main diagonal, which, together with the offdiagonal elements, characterise mutual coupling in the 


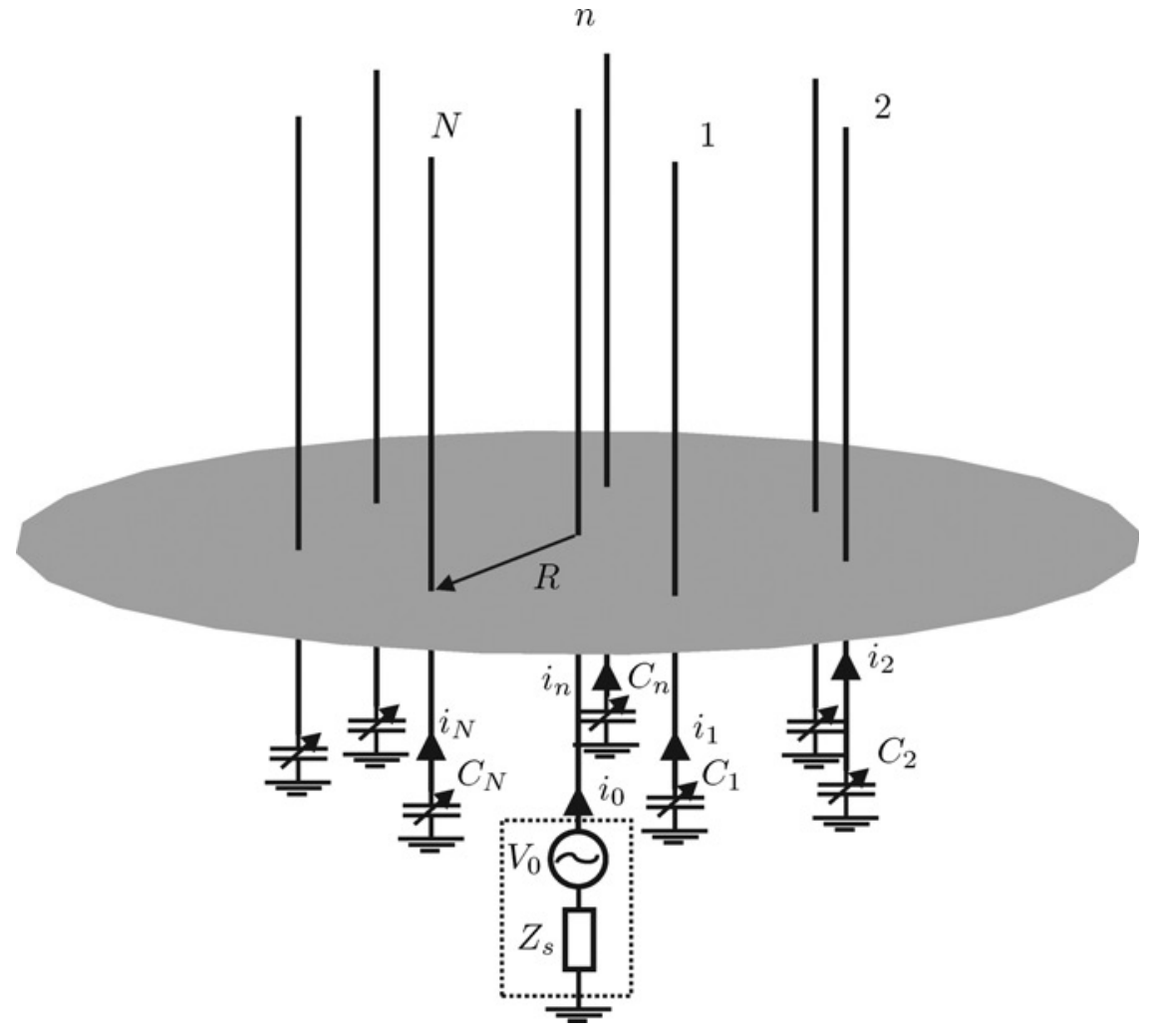

Figure 1 Uniform circular antenna array with an excited centre element

antenna system

$$
\boldsymbol{Z}=\left(\begin{array}{ccccc}
Z_{00}+Z_{s} & Z_{01} & Z_{02} & \ldots & Z_{0 N} \\
Z_{10} & Z_{11} & Z_{12} & \ldots & Z_{1 N} \\
\vdots & \vdots & \vdots & & \vdots \\
Z_{N 0} & Z_{N 1} & Z_{N 2} & \ldots & Z_{N N}
\end{array}\right)
$$

$\boldsymbol{X}$ is a diagonal matrix containing the applied reactances

$$
\boldsymbol{X}=\operatorname{diag}\left[0, x_{1}, x_{2}, \ldots, x_{N}\right]
$$

Splitting the effect of the centre element from that of the UCA elements, the radiation pattern of the ESPAR is expressed in terms of the currents obtained in (3)

$$
\begin{gathered}
\boldsymbol{F}_{\text {tot }}(\theta, \phi)=i_{0} \boldsymbol{F}_{0}(\theta, \phi)+\sum_{n=1}^{N} i_{n} \boldsymbol{F}_{n}(\theta, \phi) \\
=i_{0} \boldsymbol{F}_{0}(\theta, \phi)+\sum_{n=1}^{N} i_{n} \boldsymbol{F}_{1}\left(\theta, \phi-(n-1) \frac{2 \pi}{N}\right)
\end{gathered}
$$

where $\boldsymbol{F}_{n}(\theta, \phi)$ is the radiation pattern of the individual antenna element $n$ with the other antenna terminals being open-circuited. As neighbouring elements might affect the radiation pattern, $\boldsymbol{F}_{n}(\theta, \varphi)$ may differ from the pattern of the stand-alone antenna element. These are obtained through simulation or can be calculated directly from experimental data. In (7) we made use of the circular symmetry of the ESPAR. From (3) and (6), it is clear that mutual coupling, as described by the impedance matrix, has a large impact on the radiation pattern, which is exactly what we are trying to exploit.

\section{Radiation pattern optimisation - $\mathrm{ACO}$}

ACO is a probabilistic optimisation strategy, based on the natural behaviour of large groups of ants. Optimisation algorithms relying on natural principles, such as ACO and Particle Swarm Optimisation [9], are powerful methods that can be implemented in electromagnetic applications. Whereas the behaviour of individual ants is subject to a few basic rules, the collective behaviour exhibits highly intelligent and organised properties [10]. This is evident in the food foraging behaviour of real-life ant colonies. Reallife ants use pheromones to attract other ants to paths along which they have a high chance of finding the food. In the process, the attracted ants dispense their own pheromones, which attracts more ants. This is a positive feedback process that quickly leads to the entire colony finding an optimal path to and from the food source.

In an ACO algorithm, virtual agents called ants each create a possible solution for an optimisation problem in every iteration [5]. These are then evaluated in terms of a cost function. The best solutions are retained, or at least reinforced by applying pheromones, which are used during the next iterations to influence the solution construction 
step by the ants. Hence, better solution components are more likely to be included in the construction step by the ants in the next iteration. This mechanism exhibits a similar positive feedback process as visible in real-life foraging ants. As a result, the algorithm quickly converges to an optimal solution. As the original ACO was developed for combinatorial problems, it operates on a set of discrete parameters. However, as already mentioned in [5], ACO is a versatile approach, that is, it lends itself well for extension to the optimisation of continuous parameters, such as phase or impedance. Continuous search space $\mathrm{ACO}$ is covered in [11].

In this work, we implement a continuous ACO algorithm for ESPAR beamforming purposes. Beamforming is performed by carefully tuning adjustable reactive loads $\left(x_{1}, x_{2}, \ldots, x_{N}\right)$, which terminate the UCA elements. The currents $\left(i_{1}, i_{2}, \ldots, i_{N}\right)$ induced in the antenna elements are described by (3) and depend on the reactive loads and mutual coupling in the array configuration. From (6) it is possible to determine the beampattern $\boldsymbol{F}_{\text {tot }}(\theta, \varphi)$, which is non-linearly dependent on the reactive loads. This beampattern has to fulfil some demands. In order to compute how well a beampattern fulfils the required demands, a quality of solution is calculated by means of a cost function (8).

$$
\operatorname{cost}(\boldsymbol{X})=\exp \left[\sum_{k=0}^{K-1} \max \left(I(k) G\left(\theta_{k}, \phi_{k}, \boldsymbol{X}\right), I(k) G_{\text {goal }}\left(\theta_{k}, \phi_{k}\right)\right)\right]
$$

where $G(\theta, \phi, X)=2 \pi\left|\boldsymbol{F}_{\text {tot }}(\theta, \varphi)\right|^{2} / Z_{\mathrm{c}} P_{\mathrm{t}}$ is the established antenna power gain in the direction $(\theta, \varphi)$ for a given $X, K$ is the total number of optimisation constraints and $I(k)$ is an indicator function, which is 1 for a goal to be minimised and -1 for a goal to be maximised. $Z_{\mathrm{c}}$ is the free space impedance and $P_{\mathrm{t}}$ is the total available power at the antenna terminals. Since (3) relates the voltage and the currents through the antenna terminals for a given set of reactances $\boldsymbol{X}$, this power can easily be calculated for every evaluation of the cost function. In this work we view the optimisation as consisting of one antenna gain goal $G_{\text {goal }}\left(\theta_{0}, \phi_{0}\right)=\infty$ that is to be optimised under the constraints $G_{\text {goal }}\left(\theta_{k}, \phi_{k}\right)_{k \neq 0}$. These constraints signify a maximisation or a minimisation, depending on the sign $I(k)$. As long as the constraint goal $G_{\text {goal }}\left(\theta_{k}, \phi_{k}\right)_{k \neq 0}$ is met, the $\max (\cdot, \cdot)$ operator shuts down the maximisation, respectively minimisation, of the constraint in question by saturating the value to the constraint goal value. The proposed cost function gives a lot of flexibility for achieving the main goal $G_{\text {goal }}\left(\theta_{0}, \phi_{0}\right)$ and allows for a nearly infinite combination of different optimisation scenarios.

An arbitrary set of reactances result in a beampattern where the quality of solution $\operatorname{cost}(\boldsymbol{X}) \geq \operatorname{cost}_{\text {ideal }}$. So the problem boils down to the optimisation of $\boldsymbol{X}$ in order that the cost function is minimised. As in classic ACO algorithms, ants construct new solutions incrementally. We define a solution as a specific realisation of the $N$ reactances $\boldsymbol{X}$. In each timestep $t$, each ant creates one realisation of $\boldsymbol{X}$. For each solution component $x_{n, t}$, this is the reactance $x_{n}$ at timestep $t$, they make use of the pheromone levels $f^{n, t}(x)$ to probabilistically influence their choice. The corresponding probability mass function for choosing the parameter $x_{n}$ at construction step $n$ during timestep $t$ is

$$
P^{n, t}(x)=\frac{f^{n, t}(x)}{\sum f^{n, t}(x)}
$$

where the summation is performed over all possible values of $x_{n}$. This pheromone concept works fine for combinatorial problems such as travelling salesman problem, but an extension is needed for continuous search spaces, such as for reactances or phases. In [11] a Gaussian mixture model is proposed to model the probability density function (pdf) $p^{n, t}(x)$ (Fig. 2b), replacing $P^{n, t}(x)$ (Fig. 2a) in the case of continuous search spaces. The probability density function for choosing $x_{n}$ is now given by

$$
p^{n, t}(x)=\frac{f^{n, t}(x)}{\int f^{n, t}(x) \mathrm{d} x}
$$

where the integration is performed over the interval of allowable $x_{n}$ values. By means of the pdfs, $M$ ants will construct $M$ realisations of $\boldsymbol{X}$ resulting in $\left(x_{1, t}^{m}, x_{2, t}^{m}, \ldots, x_{N, t}^{m}\right)(m=1, \ldots, M)$.

Once the constructed solutions are evaluated using the cost function (8), the pheromone pdfs are updated for the next iteration step $t+1$

$$
f^{n, t+1}(x)=(1-\rho) f^{n, t}(x)+\Delta f^{n, t}(x)
$$

where $\rho$ is a parameter that describes the evaporation of the pheromone trail. The change in pheromone concentration between time steps is given by

$$
\Delta f^{n, t}(x)=\sum_{m=1}^{M} \frac{Q}{\operatorname{cost}\left(X_{t}^{m}\right)} \frac{1}{\sqrt{2 \pi} \sigma_{n, t}} \mathrm{e}^{-\left(x-x_{n, t}^{m}\right)^{2} / 2 \sigma_{n, t}^{2}}
$$

This means the pheromone concentration is changed by adding a normal distribution with a standard deviation $\sigma_{n, t}$ around solution component $x_{n, t}^{m}$. This use of normal distributions in continuous ACO was first proposed in [11] and can be considered an application of the principle of maximum entropy. The scaling factor $Q / \operatorname{cost}\left(\boldsymbol{X}_{t}^{m}\right)$ of the normal distribution depends on the cost of the solution. Good solutions will get a higher weight factor, meaning they will represent a greater probability density in (10), which will introduce the desired positive feedback towards good solutions. Notice that the normal distribution in the continuous implementation reduces to a delta function in a discrete implementation. The 

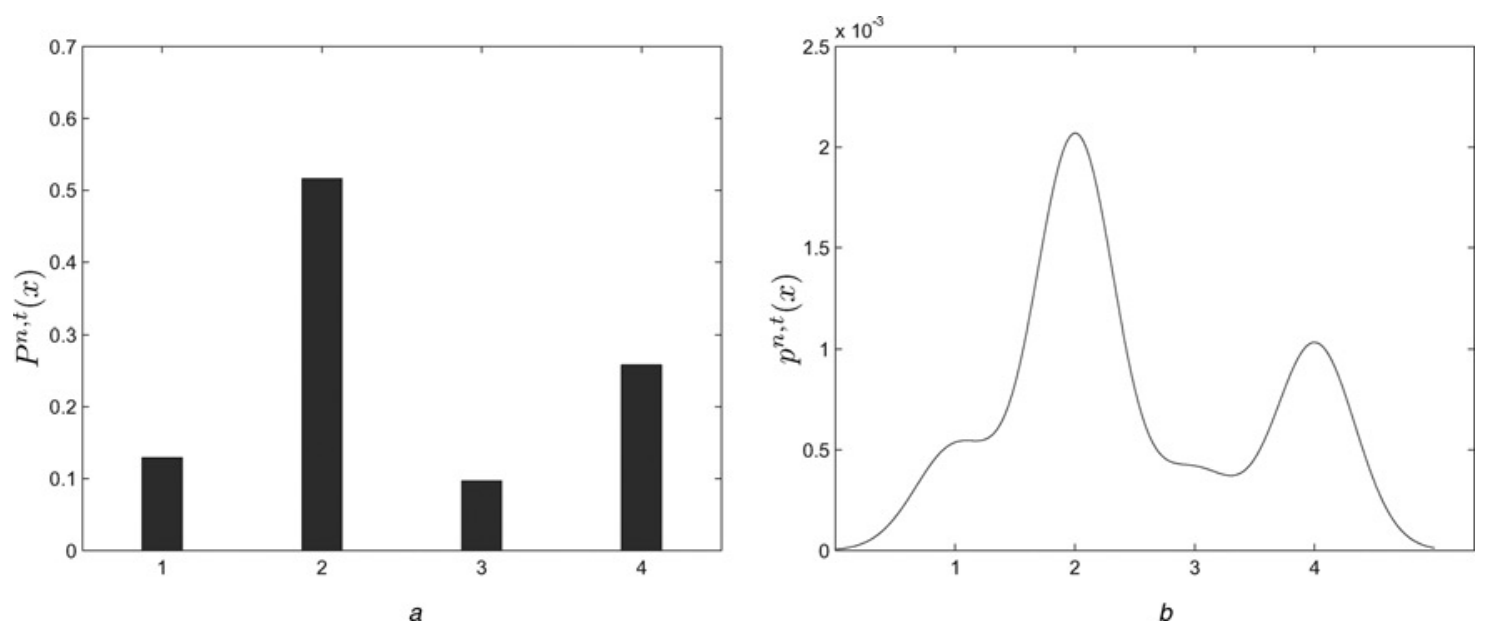

Figure 2 Comparison between search spaces

a Four-choice discrete search space

$b$ Corresponding continuous search space

standard deviation $\sigma_{n, t}$ adds an extra degree of freedom to the continuous implementation of the algorithm, which we will call the pheromone width from now onwards. It is easy to see how the value of $\sigma_{n, t}$ influences the solution construction step. A large pheromone width results in drastic changes in solution component, whereas a small pheromone width results in small changes, that is, solution components that differ very little from already explored ones [10]. This behaviour is analogue to the temperature parameter in simulated annealing class algorithms. We therefore propose a similar usage of the pheromone width parameter. As the algorithm converges to an optimum, we let the pheromone width cool down, that is, decrease with some cooling rate factor. As in [11], we opt for the cool-down formula (13)

$$
\sigma_{n, t}=\max \left(\frac{\max \left(x_{1, \ldots, M}^{n, t}\right)-\min \left(x_{1, \ldots, M}^{n, t}\right)}{\sqrt{t}}, \epsilon\right)
$$

with $\epsilon$ a pheromone width threshold. Note that, apart from cooling down with a constant factor $1 / \sqrt{t}, \sigma_{n, t}$ depends on the distance between the choices $x_{n, t}$ made by the $M$ ants, which adds to robustness against local optima. Similar parameters are one of the defining characteristics of ES algorithms [6]. In ES, evolvable parameters, called 'endogenous', are used. These are crucial in the selfadaptive mutation paradigm of ES. In a similar way, but without altering the defining characteristics of an ACO algorithm, the proposed technique introduces this selfadaptiveness into to continuous ACO framework. ES algorithms use recombination and mutation, in a broader sense than genetic optimisation, whereas the proposed ACO algorithm uses the inter-agent communcation through pheromones as a sort of informed 'recombination' operator.

\section{Experiments and results}

The proposed algorithm was implemented by combining superposition, (3) and (6), with the ACO technique. The technique was optimised for speed and predictability, to provide fast convergence. We now demonstrate the algorithm and highlight some important aspects.

\subsection{Implementation issues}

In this section, we apply the algorithm to a UCA with radius $\lambda$ consisting of 16 quarter-wavelength monopole antennas operating at $2.45 \mathrm{GHz}$.

4.1.1 Discrete demands: We start off with a discrete optimisation demand, maximal antenna gain in the direction of azimuth angle $\phi=0^{\circ}$. Our first experiment aims to determine whether a direct optimisation of the involved impedances $\boldsymbol{X}$ is the best option. This optimisation was performed using variable reactances consisting of a fixed inductance of $5 \mathrm{nH}$ in series with a variable capacitance between 0.1 and $10 \mathrm{pF}$. The algorithm was implemented using 10 ants, a scaling quality factor $Q=1$, pheromone trail evaporation factor $\rho=0.125$ and pheromone width threshold $\epsilon=\left(\max \left(x_{1, \ldots, M}^{n, t}\right)-\min \left(x_{1, \ldots, M}^{n, t}\right)\right) / 2048$. We view the optimisation problem as the search for optimal phase steering of a phased array antenna. This point of view is possible through the consideration of the impedances $\boldsymbol{X}$ as phase shifting devices. We can describe the array elements from Fig. 1 by the circuit model in Fig. 3, where we have used superposition to combine the mutual coupling-induced voltages into a single voltage $V^{n}$. The point of interest then becomes the influence of the added reactance $j x_{n}$ on the current $i_{n}$ flowing through the antenna terminals. 


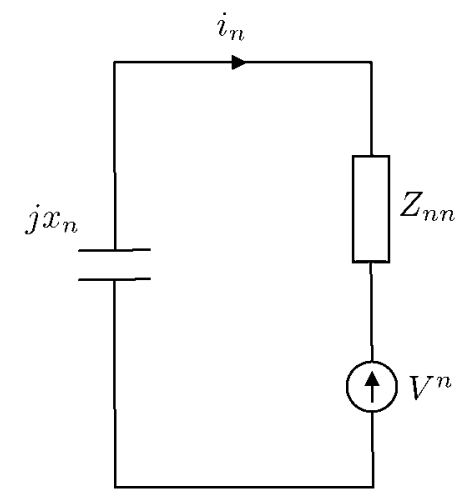

Figure 3 Circuit equivalent of array element $n$

The relative phase shift $f_{n}$ with regard to $V^{n}$ (Fig. 3), introduced by a chosen reactance $x_{n}$, is easily found (where $\left.Z_{n n}=R_{n n}+j X_{n n}\right)$

$$
\begin{aligned}
& f_{n}=-\arctan \left(\frac{x_{n}+X_{n n}}{R_{n n}}\right) \\
& x_{n}=c \in\left[x_{\min }, x_{\max }\right]
\end{aligned}
$$

This is a highly non-linear characteristic. This non-linearity has an undesirable effect on the search space. The sensitivity to changes in the optimisation parameter $f_{n}$ is highly dependent on the value of $x_{n}$. This conflicts with the notion of pheromone width: for a fixed $\sigma_{n, t}$, the expected change in current $i_{n}$ by adapting the phase $f_{n}$ is greater for small values of $x_{n}$, than for a large value of $x_{n}$. The problem here is that the idea behind pheromone width is to capture the allowed change in optimisation result, that is, the sensitivity, during the next construction step. To solve this problem, we propose a new approach for the optimisation problem. Instead of performing optimisation in reactance values, as in (14), we will operate in a multidimensional phase space and calculate the corresponding capacitance afterwards

$$
f_{n}=c \in\left[-\arctan \left(\frac{x_{\min }+X_{n n}}{R_{n n}}\right),-\arctan \left(\frac{x_{\max }+X_{n n}}{R_{n n}}\right)\right]
$$

Both approaches were compared in Fig. 4. Here, a $\lambda / 4$ radius, six-element UCA with centre element, operating at $2.484 \mathrm{GHz}$, was optimised for maximal main beam gain. The reactive loads were allowed to vary between 0.75 and $2 \mathrm{pF}$. The algorithm used 10 ants, a scaling quality factor $Q=1$, pheromone trail evaporation factor $\rho=0.125$ and pheromone width threshold $\epsilon=\left(\max \left(x_{1, \ldots, M}^{n, t}\right)-\min \right.$ $\left.\left(x_{1, \ldots, M}^{n, t}\right)\right) / 2048$. The dotted line represents convergence of the algorithm that optimises reactances (14). The full line represents the convergence of the algorithm that optimises phases directly, chosing values within the limits defined in (15). The achieved solution is much better for the phase optimisation, which suggests that the reactance optimising algorithm converges to a local optimum. The parameter $\sigma_{n, t}$, the pheromone width (Section 3), which smoothes the

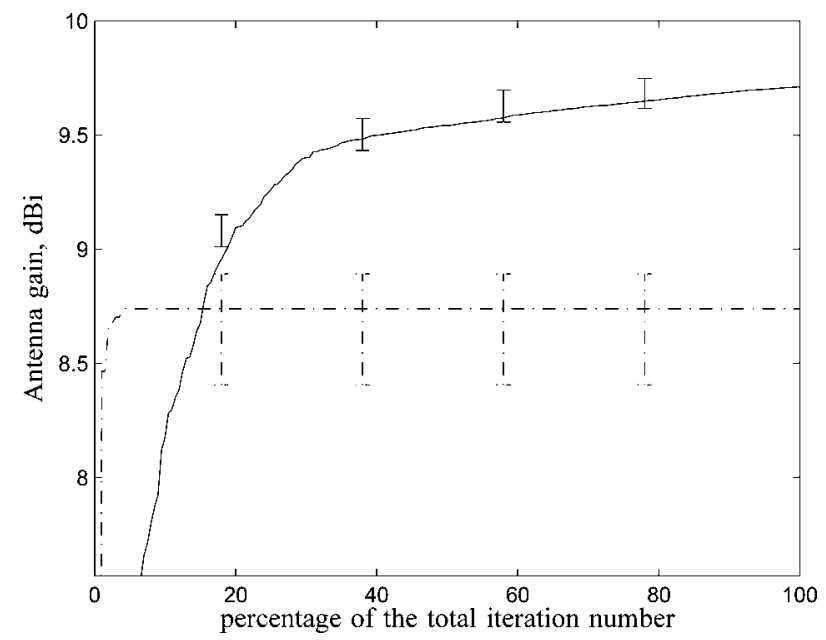

Figure 4 Convergence of the optimisation algorithm: optimising the reactances (dotted line), optimising the phases (full line)

The mean best solution is shown. Also shown are bars depicting the interquartile range (IQR) of the best attained solutions at 20 $40,60,80$ of the total iteration number

search space, should have prevented this. This is explained through the highly non-linear influence of the reactance on the cost function. The same $\sigma_{n, t}$ results in very different sensitivity of the cost function to the resulting changes in reactance, depending on the value of the reactance, that is, the position of the pheromone distribution in the search space. The effect of $\sigma_{n, t}$ therefore varies greatly within the search space. Performing optimisation on the phases directly (15), linearises the search space somewhat, thereby reducing the influence of the absolute position of the pheromone distribution in the search space on the sensitivity of the cost function to $\sigma_{n, t}$. With this alteration, the pheromone width $\sigma_{n, t}$ works as it should, smoothing the search space in order to avoid premature convergence to local optima. This is evidenced by the superior solution indicated by the full line in Fig. 4.

4.1.2 Continuous demands: Beamforming requirements are very often provided as a set of continuous demands on the radiation pattern. As digital signal processing inevitably implies a discretisation of the radiation pattern, one also has to decide which sampling frequency to use for the continuous demands. This is important because there is a linear relationship between the execution time and the sampling frequency. In [12], it is proven that the radiation pattern is a band limited signal for the azimuth angle $\phi$. In [13] this has been used to propose a proportional relationship between the minimum required sampling frequency $f_{\mathrm{s}}$ and the electrical size $k_{0} R$ of a uniform circular array

$$
f_{\mathrm{s}}=\frac{k_{0} R}{\pi}
$$

$k_{0} R / \pi$ is equivalent to the Nyquist rate. Simply using the 


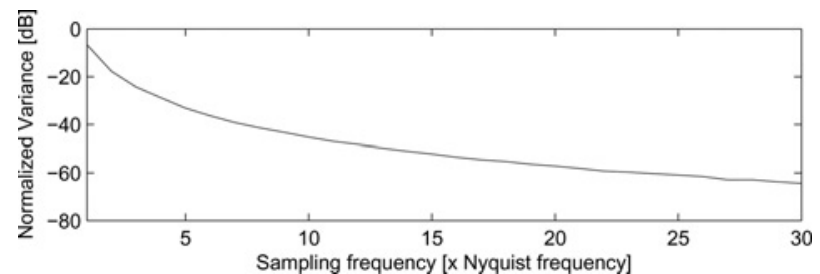

Figure 5 Evolution of the normalised quantisation error variance as the radiation pattern sampling frequency increases

Nyquist rate to discretise a continuous demand on the radiation pattern is not sufficient. This will still result in overshoot: According to the Fourier theorem, any periodic function (such as the antenna gain pattern) can be expressed as a series of sines. Since the bandwidth is limited, there will be a sine of highest frequency, this one will be sampled with the least (relative to the sines of lower frequency). If there are any problems regarding the sampling of the antenna gain pattern, it will be with this sine. A continuous sine always reaches its maximum in between sample points. Monte Carlo simulations show that there is a clear relationship between the sampling rate and the expected overshoot. This is shown in Fig. 5. The figure shows the evolution of the variance between the largest sample point value and the maximum of the continuous sine. The variance of the quantisation error on the cost function has already dropped $35 \mathrm{~dB}$ if we use five times the Nyquist frequency. A comparison between optimisation with discretisation using the Nyquist frequency and discretisation using five times the Nyquist frequency is shown in Figs. 6 and 7 where, in addition to creating a main beam along $0^{\circ}$, the algorithm has attempted to suppress the side lobe level by $20 \mathrm{~dB}$ for azimuth angles between $\phi=90^{\circ}$ and $135^{\circ}$.

This optimisation was performed for the 16-element quarterwavelength monopole UCA with array radius $\lambda$ fed by

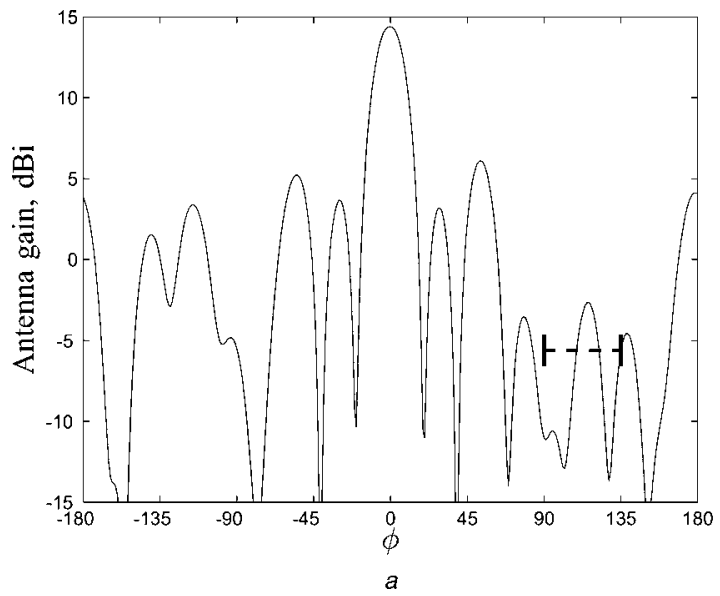

phase-controlled ideal voltage sources at $2.45 \mathrm{GHz}$. Again, the algorithm was implemented using 10 ants, a scaling quality factor $Q=1$, pheromone trail evaporation factor $\rho=0.125$ and pheromone width threshold $\epsilon=$ $\left(\max \left(x_{1, \ldots, M}^{n, t}\right)-\min \left(x_{1, \ldots, M}^{n, t}\right)\right) / 2048$. The radius of the array is $\lambda$, so the Nyquist frequency is equal to 2 samples/radian.

\subsection{Matching requirements}

For the optimisation of the 16-element quarter-wavelength monopole UCA with array radius $\lambda$ fed by phase-controlled ideal voltage sources at $2.45 \mathrm{GHz}$, we allowed reactance to vary between 70.47 and $-572.64 \Omega$. This is roughly equivalent with a fixed series inductance of $5 \mathrm{nH}$ with capacitances varying between 0.1 and $10 \mathrm{pF}$. The optimisation is discussed in Section 4.4. In this search space, antenna impedance varies between 68.00-j26.17 and 102.27-j103.46 $\Omega$. This means mismatch factors vary between 0.6035 and 0.9309 and standing wave ratio (SWR) between 4.4 and 1.7 when matching to a source impedance of $50 \Omega$. It is clear that the proposed method of steering an ESPAR influences the impedance matrix in a very real way. The antenna input impedance will drift while steering, resulting in a change of the mismatch factor and SWR. The impedance matrix for one configuration of reactances is constantly calculated in the proposed framework. Through this, transmitted power, mismatch factor and SWR are calculated for every ant in every cycle. It is therefore possible to include matching requirements in the cost function to exclude solutions that are not sufficiently matched.

\subsection{Performance}

4.3.1 Execution speed: The combination of an electromagnetic description of the ESPAR in full detail and the ACO technique is optimised for speed and predictability, to provide fast convergence. It has been compared to the free software 4NEC2's [14] genetic

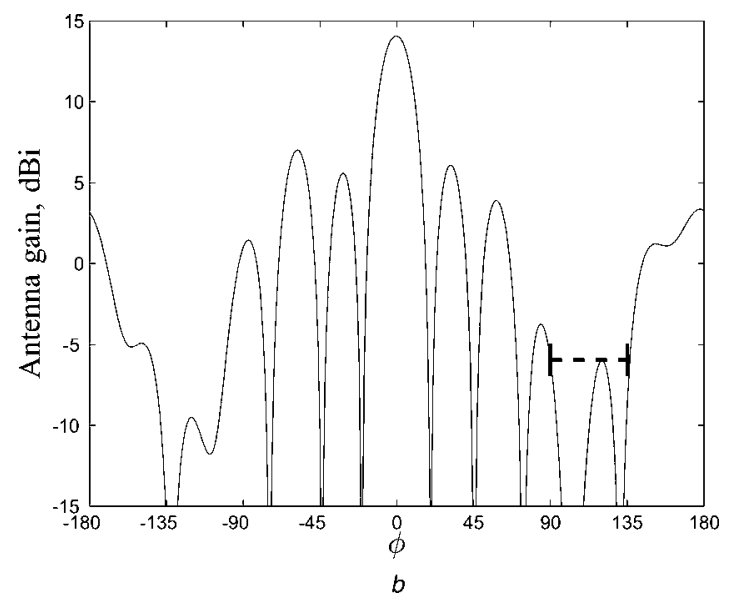

Figure 6 Gain of the optimised ESPAR

a Comparison between optimisation using the Nyquist rate

$b$ Comparison between optimisation using five times the Nyquist rate

The $20 \mathrm{~dB}$ suppression limit is denoted by the dotted line 

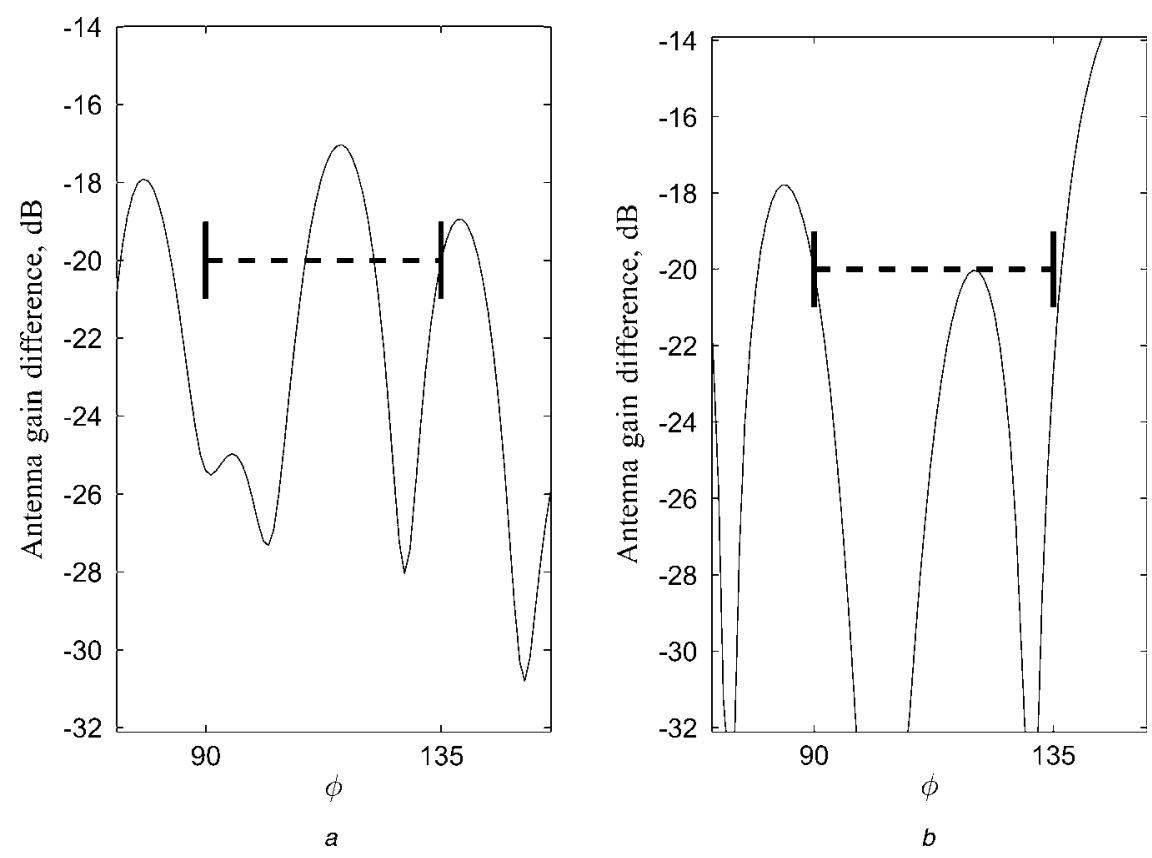

Figure 7 Cropout from Fig. 6

The gain of the optimised ESPAR in the interval $\varphi \in\left[90^{\circ}, 135^{\circ}\right]$

a Comparison between optimisation using the Nyquist rate

$b$ Comparison between optimisation using five times the Nyquist rate

algorithm optimiser for solution quality as a function of the number of iterations. The genetic algorithm had a population size of 30 , crossover probability of $70 \%$ and mutation probability of $5 \%$ the proposed ACO algorithm had a 10 ants, a scaling quality factor $Q=1$, pheromone trail evaporation factor $\rho=0.125$ and pheromone width threshold $\quad \epsilon=\left(\max \left(x_{1, \ldots, M}^{n, t}\right)-\min \left(x_{1, \ldots, M}^{n, t}\right)\right) / 2048$. The optimisation problem was the same as in Section 4.1.1. The convergence characteristics are shown in Fig. 8. Both algorithms converge in a remarkably similar number of iterations, although the population of the proposed algorithm was much smaller. This is an indication for the potential of the proposed method. There is, however, a huge difference in execution time between the genetic optimisation and the proposed method. Whereas the genetic optimiser uses the NEC full-wave solver for evaluating each gene during every iteration, which causes that optimisation process to take several hours, the proposed algorithm uses the superposition technique explained in Section 2, which takes only fractions of a second (98 ms on a $2.1 \mathrm{GHz}$ AthlonXP processor). In that sense the proposed algorithm could be implemented for real-time purposes. This comparison proves a clear advantage when using the superposition technique from Section 2 instead of when evaluating a full-wave solver for every ant/gene in every generation.

4.3.2 Comparison to other techniques: In [2], an analytical approach was adopted for the same optimisation problem. In this example, a six-element UCA was used with radiators located at radius $\lambda / 4$ around the centre element. The added capacities in [2] were allowed to vary within the range of $0.1-10 \mathrm{pF}$, the operating frequency is $2.484 \mathrm{GHz}$. This analytical approach optimises the electric field. The electric field, however, is a less suitable quality measure than the antenna gain for an antenna system, as the comparison with the proposed method in Fig. 9 shows. The directivity is far worse in the case of electric field maximisation compared to maximisation of the antenna gain. The aim of the experiment was to maximise the main beam antenna directivity in the direction of $\varphi=0^{\circ}$. For the $\mathrm{ACO}$ algorithm, we used the same parameters as in the previous experiments.

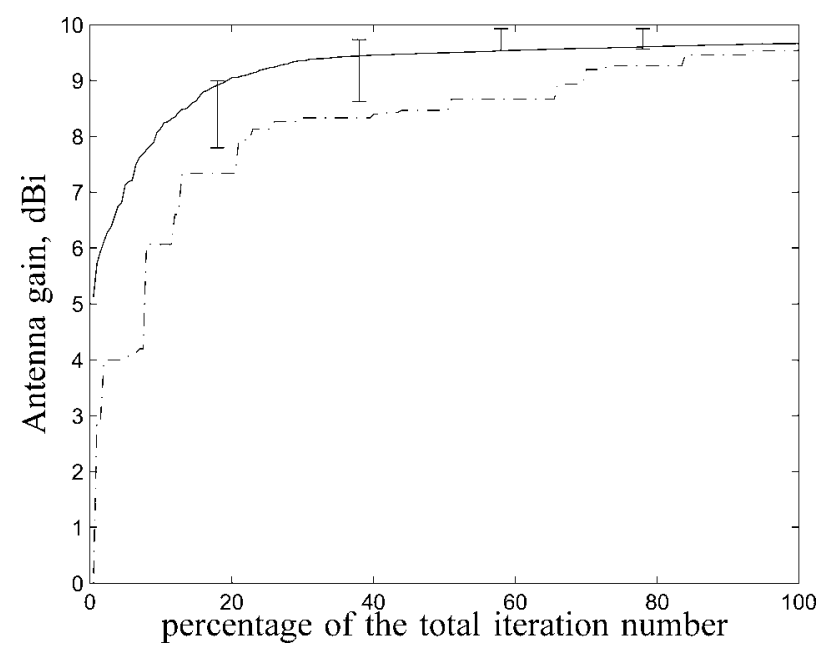

Figure 8 Convergence comparison between the proposed ACO (full line) and a genetic algorithm (dotted line), the bars depict the IQR 


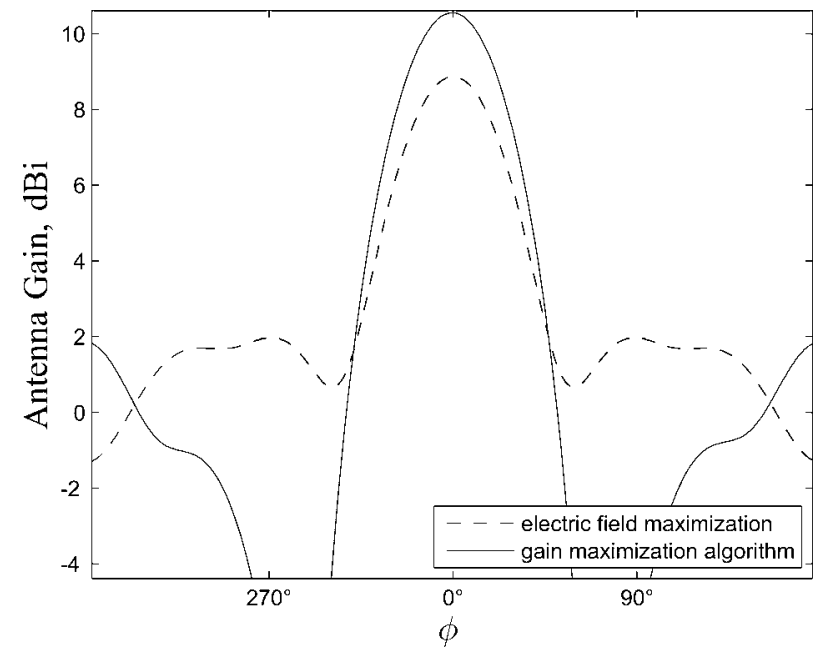

Figure 9 Comparison between electric field maximisation, the analytical approach from [2] (dotted line) and antenna gain maximisation, our proposed technique (full line)

\subsection{Antenna design experiment}

We now apply the algorithm again to the 16-element UCA of monopole elements. Fig. 10 shows the result of the antenna gain maximisation problem in the direction of $\phi=0^{\circ}$ (in the direction of a UCA element) as well as for a main beam steered along $\phi=11.25^{\circ}$ (direction in between two UCA elements). This optimisation was performed using variable reactances consisting of a fixed inductance of $5 \mathrm{nH}$ in series with a variable capacitance between 0.1 and $10 \mathrm{pF}$. Again, the algorithm was implemented using 10 ants, a scaling quality factor $Q=1$, pheromone trail evaporation factor $\sigma=0.125$ and pheromone width threshold $\epsilon=\left(\max \left(x_{1, \ldots, M}^{n, t}\right)\right.$ $\left.-\min \left(x_{1, \ldots, M}^{n, t}\right)\right) / 2048$. The difference in the main lobe gain in the two situations is $0.3 \mathrm{~dB}$. Aside from the obvious main

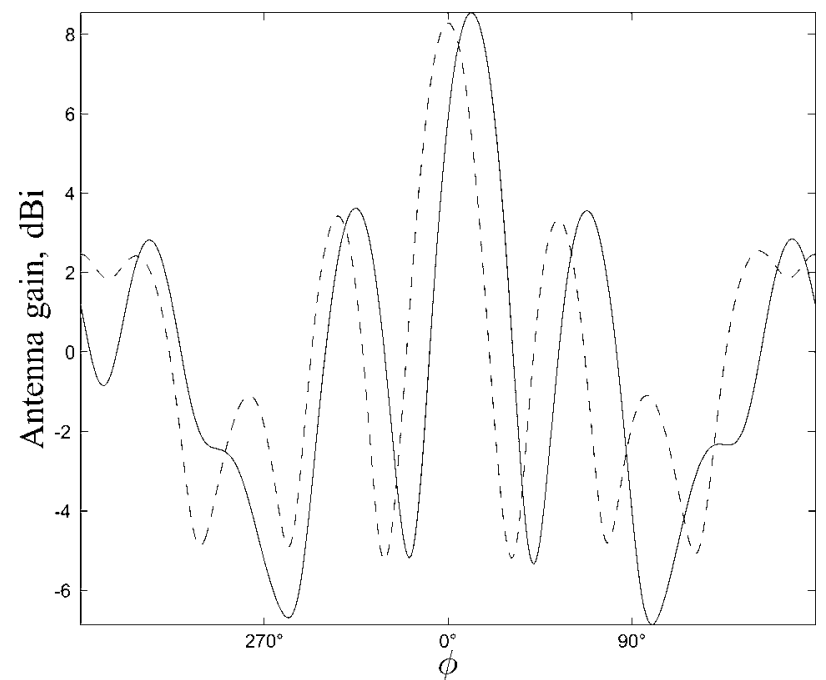

Figure 10 Result from gain maximisation for the 16element ESPAR, main beam direction $\phi=0^{\circ}$ (dotted line) and main beam direction $\phi=11.25^{\circ}$ (full line)

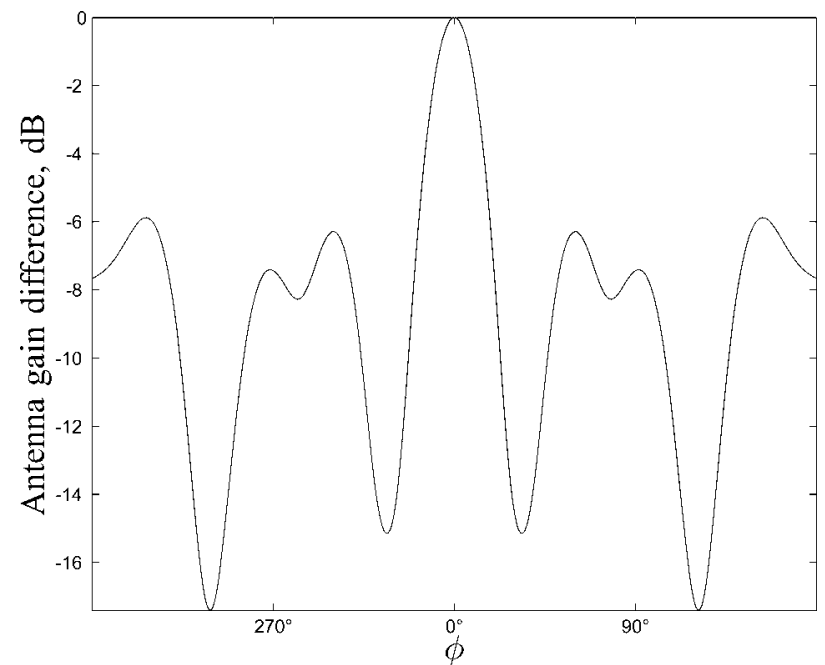

Figure 11 Result of the gain maximisation/side lobe suppression for the 16-element UCA

beam directivity, two large side lobes come into view. These are suppressed by only $4.5 \mathrm{~dB}$ with respect to the main beam. Using the technique sketched in 4.1.2, an attempt was made to suppress these side lobes by $10 \mathrm{~dB}$. The best attainable side lobe suppression, without significantly comprimising the main beam gain and/or causing other side lobes, is found to be $6.5 \mathrm{~dB}$ (see Fig. 11). The optimised impedance values are shown in Table 1.

Table 1 List of impedance values at the 16-element UCA for the optimised problem of Section 4.4

\begin{tabular}{|l|c|}
\hline Antenna Azimuth (deg) & Impedance value $(+5 \mathrm{nH})(\mathrm{pF})$ \\
\hline 0 & 1 \\
\hline 22.5 & 0.8 \\
\hline 45 & 0.2 \\
\hline 67.5 & 1.3 \\
\hline 90 & 0.3 \\
\hline 112.5 & 9.8 \\
\hline 135 & 9.3 \\
\hline 157.5 & 1.3 \\
\hline 180 & 0.8 \\
\hline 202.5 & 1.5 \\
\hline 225 & 9.0 \\
\hline 247.5 & 9.3 \\
\hline 270 & 0.3 \\
\hline 292.5 & 1.3 \\
\hline 315 & 0.2 \\
\hline 337.5 & 0.8 \\
\hline
\end{tabular}




\section{Conclusion}

In this paper, it has been demonstrated that $\mathrm{ACO}$ is a viable technique for use in antenna optimisation problems. It withstands a direct comparison with genetic algorithms. It was also demonstrated that the necessary calculations for variable reactance beamforming are possible, in real-time, and for a very large number of different radiation pattern and matching restrictions. The number of different optimisation scenarios can be nearly infinite, which is very important when comparing with other methods (optimisation and/or other means), which would have to be used off-line and have their results stored in a ROM of limited capacity.

\section{References}

[1] SUN C., HIRATA A., OHIRA T., KARMAKAR N.C.: 'Fast beamforming of electronically steerable parasitic array reflector antennas: theory and experiment', IEEE Trans. Antennas Propag., 2004, 52, (7), pp. 1819-1832

[2] SCHAER B., RAMBABU K., BORNEMANN J., VAHLDIECK R.: 'Design of reactive parasitic elements in electronic beam steering arrays', IEEE Trans. Antennas Propag., 2005, 53, (6), pp. $1998-2003$

[3] CHENG J., HASHIGUCHI M., LIGUSA K., OHIRA T.: 'Electronically steerable parasitic array radiator antenna for omni- and sector pattern forming applications to wireless ad hoc networks', IEE Proc.-Microw. Antennas Propag., 2003, 150, (4), pp. 203-208

[4] HARRINGTON R.F.: 'Reactively controlled directive arrays', IEEE Trans. Antennas Propag., 1978, 26, (3), pp. 390-395

[5] DORIGO M., MANIEZZO V., COLORNI A.: 'Ant system: optimization by a colony of cooperating agents', IEEE Trans. Syst., Man, Cyberneti., 1996, 26, (1), pp. 1-13
[6] BEYER H.G., SCHWEFEL H.P.: 'Evolution strategies: a comprehensive introduction', Natural Comput., 2002, 1,

(1), pp. 3-52

[7] SCHLUB R., LU J., OHIRA T.: 'Seven-element ground skirt monopole ESPAR antenna design from a genetic algorithm and the finite element method', IEEE Trans. Antennas Propag., 2005, 53, (6), pp. 1998-2003

[8] Boeringer D.W., WeRner D.H.: 'Particle swarm optimisation versus genetic algorithms for phased array synthesis', IEEE Trans. Antennas Propag., 2004, 52, (3), pp. 771-779

[9] ROBINSON J., RAHMAT-SAMII Y.: 'Particle swarm optimisation in electromagnetics', IEEE Trans. Antennas Propag., 2004, 52, (2), pp. 397-407

[10] COLEMAN C.M., ROSS J.E.: 'Investigation of simulated annealing, ant-colony optimization, and genetic algorithms for self-structuring antennas', IEEE Trans. Antennas Propag., 2004, 52, (4), pp. 1007-1014

[11] SOCHA K.: 'ACO for continuous and mixedvariable optimization', Ant colony, optimization and swarm intelligence, vol. 3172 (Springer, Berlin, 2004), pp. $25-36$

[12] ROGIER H., BONEK E.: 'Analytical spherical-mode-based compensation of mutual coupling in uniform circular arrays for direction-of-arrival estimation', Int. J. Electron. commun., 2006, 60, (2), pp. 176-189

[13] Aelterman J.: 'Ontwerp van een directieve draadantenne met parasitaire stralers via een optimalisatie-algoritme gebaseerd op het gedrag van mierenkolonies', MS thesis, Ghent University, 2007

[14] VOORS A.: 'NEC based antenna modeller and optimizer', http://home.ict.nl/arivoors/ 